\title{
Rompiendo barreras en el pensamiento creativo desde la enseñanza media
}

\section{Resumen}

Se describen aquí los esfuerzos en los que se han involucrado algunas instituciones de enseñanza privadas y públicas como el Massachusetts Institute of Technology, el Rhode Island School of Design o escuelas privadas como NuVu en Cambridge para incorporar el arte en la enseñanza de materias troncales en las que se incluyen ciencias, tecnología, ingeniería y matemáticas (STEM por sus siglas en inglés). Estas y otras instituciones están haciendo grandes logros para proveer a estudiantes de enseñanzas medias la capacidad para incorporar el pensamiento creativo, critical thinking, para ayudarles a ser mejores líderes en sus áreas de especialización.

Palabras clave: Pensamiento creativo, captación de estudiantes, clases de enriquecimiento, MIT, RISD, Brown University, NSTA.org, Isabella Stewart Gardner Museum.

\section{Breaking barriers from Middle and High School education through critical thinking}

\section{Abstract}

This article describes the approaches some educational institutions, private and public, are doing to incorporate Art in the traditional setting of Sciences, Technology, Engineering and Mathematics (STEM). Institutions such as the Rhode Island School of Design, Massachusetts Institute of Technology or private after schools such as NuVu in Cambridge, are making great strides in providing middle school and high school students the tools to incorporate critical thinking to help them become better industry leaders.

Keywords: critical thinking, STEAM, captación de estudiantes, clases de enriquecimiento, MIT, RISD, Brown University, NSTA.org, Isabella Stewart Gardner Museum, rapid prototyping, Educación global.

1 Jorge Paricio García es profesor en el departamento de diseño de interiores en el The Art Institute of Pittsburgh y profesor visitante en el Rhode Island School of Design, en los departamentos de Continuing Education y Diseño Industrial. Recientemente ha publicado un libro titulado Hybrid Drawing Techniques for Interior Design. 
En una fría mañana de febrero me acerco al departamento de arquitectura del Instituto de Tecnología de Massachusetts (MIT en sus siglas en inglés) en donde mantengo una conversación con Paul Pettigrew, director de admisión de estudiantes de licenciatura, desarrollo profesional y contacto con antiguos alumnos, en la escuela de arquitectura. Nuestra conversación gira entorno a cómo MIT en la actualidad intenta captar estudiantes que muestren tener aptitudes por encima de las ramas consideradas troncales, dentro del formato de estudios STEM, que en sus siglas en inglés abarca los estudios de ciencias, tecnología, ingeniería y matemáticas.

Las escuelas de educación de grado intermedio y grado superior en la última década han sufrido recortes en el presupuesto, y han volcado sus energías en ramas del estudio consideradas fundamentales a la hora de encontrar empleo. Los diferentes departamentos de educación de cada estado han promocionado esta manera de ver la educación, y sus baremos y estándares así lo reflejan. Como consecuencia de este giro en la educación, otras materias han sido paulatinamente relegadas a un segundo plano, o incluso convertidas en asignaturas optativas o fuera del horario escolar, y son llamadas eufemísticamente clases de enriquecimiento, como fotografía, pintura, dibujo artístico o técnico. Según la Asociación Nacional de Profesores de Ciencias (NSTA), este posicionamiento ayudará a los Estados Unidos a obtener una nueva generación de innovadores y líderes en la industria, a través de cursos en institutos que preparen a futuros estudiantes de universidad en diferentes ramas técnicas, como bioquímica, ingeniería, programación, tecnologías emergentes o ingeniería aeroespacial.

Sin embargo, en este ambiente de aprendizaje volcado hacia la tecnología, muchas universidades encuentran que los estudiantes que llegan del instituto tienen buena preparación técnica, pero carecen de un sentido de la exploración y la creatividad que se adquiere con un diseño curricular más amplio. La admisión en muchas universidades americanas es ya muy competitiva de por sí. Por ejemplo, el MIT, la Universidad de Stanford o Brown University, tienen un porcentaje de admisión del 7\%, y el de Harvard alcanza todavía un índice más bajo, llegando al $5 \%$, y prefieren admitir alumnos que también tengan una educación en que se incluya en pensamiento creativo.

Algunas universidades, como el Rhode Island School of Design (RISD) han contraatacado en esta carrera hacia la tecnología, y ha sugerido cambiar las siglas STEM por STEAM en la enseñanza de grado medio, en las que el Arte ha sido incluido como otra asignatura troncal. En el año 2013, la profesora y directora del departamento de Diseño de Mobiliario, Rosanne Somerson (actual presidenta de RISD) publicó "el arte de hacer con pensamiento crítico", (The Art of Critical Making) en el que se evaluaban las prácticas creativas que utiliza esta universidad en muchos de sus departamentos avanzados, en el campo de las bellas artes y el diseño gráfico, diseño industrial, diseño de mobiliario, diseño textil o arquitectura. El libro analiza muchos procesos creativos que usa esta universidad en los que se enfatiza el fabricar ideas u objetos, inventando materiales y activando o provocando experiencias que ayuden en el proceso físico de creación en tres dimensiones. 
Desde el MIT, Paul Petigrew describe el cambio de actitud de su universidad hacia un tipo de estudiante que tenga una capacidad de pensar creativa. En un ambiente muy competitivo y técnico, en el que los estudiantes tienen que estudiar una serie de clases obligatorias, como física, cálculo, química, biología y laboratorio, los alumnos tienen la libertad de escoger una tercera parte de sus asignaturas como libre elección y así se obtienen interesantes mezclas. Paul comenta que, de este espíritu de apertura a nuevas maneras de ver la educación, pueden salir grandes oportunidades.

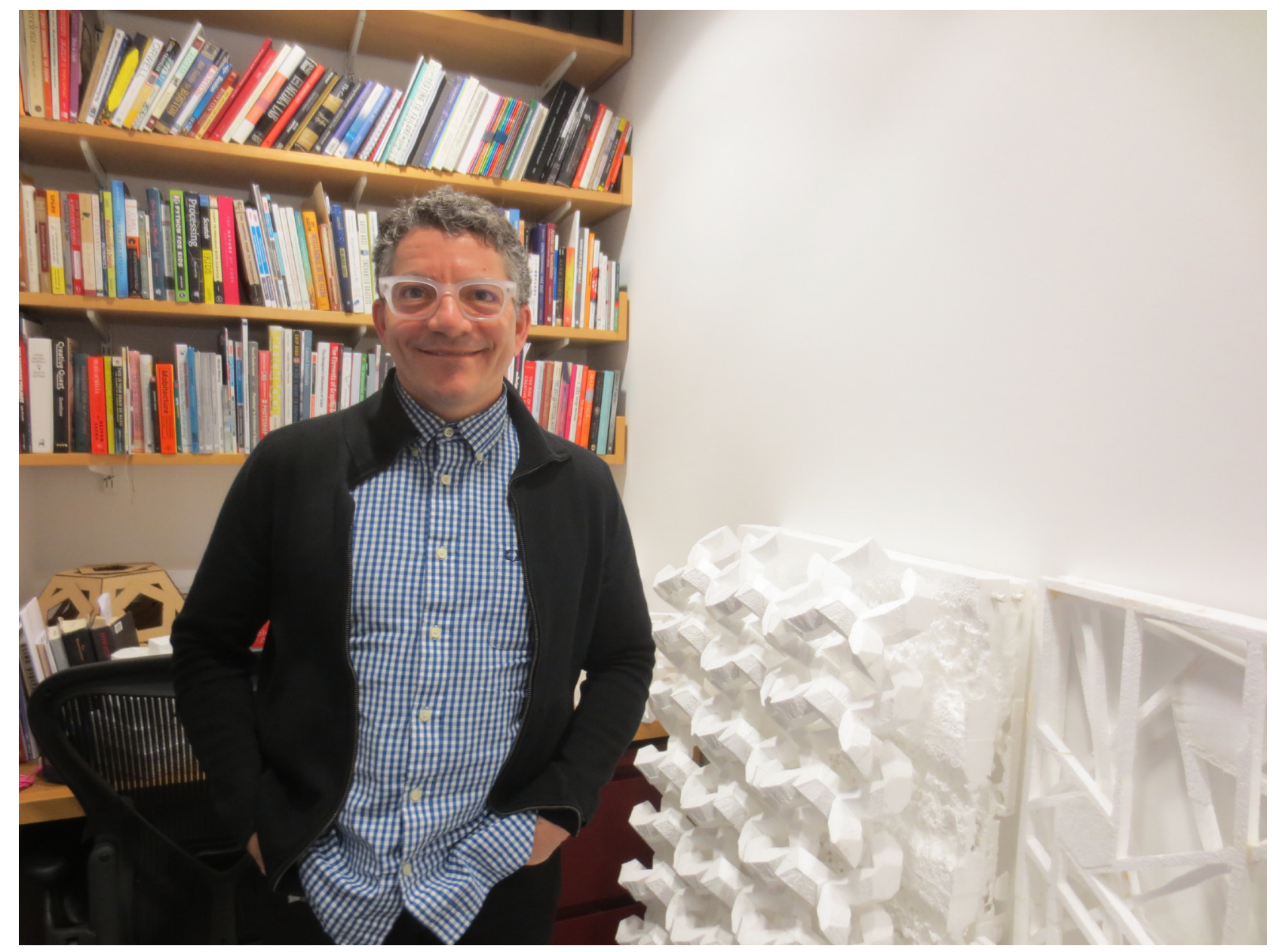

Fig. 1. Paul Pettigrew en su estudio en el MIT, Cambridge. Fotografía de Jorge Paricio.

De su departamento de Arquitectura ha visto salir a graduados en arquitectura, pero con un aprendizaje complementario en ciencias de la computación, o con una síntesis de arquitectura y química. Me cuenta el caso de Erika Anderson, que está terminando su especialidad en ingeniería mecánica con una concentración secundaria en diseño y cultura. La universidad le dio la oportunidad de publicar una revista de moda y diseño, a la vez que le ha facilitado el acceso a talleres de costura en los que experimentar con nuevos materiales textiles, que pueden cambiar de color según una serie de variables.

En esta misma línea de pensamiento, Saeed Arida, un graduado de MIT en computación y diseño, fundó hace diez años en Cambridge, Massachusetts, el estudio NuVu (en inglés se pronuncia como New View) con 
la idea de proveer a alumnos de enseñanza media una educación de calidad que no tenían en sus escuelas originales. Y así estableció convenios de colaboración con muchas escuelas locales y a nivel nacional e internacional, en la utilización de nuevas tecnologías de fabricación, pensamiento creativo en equipo, creación de prototipos rápidos y modelado, y trabajo iterativo. NuVu ofrece cursos de duración variable con la ayuda de un equipo de producción que incluye arquitectos, ingenieros y diseñadores industriales. Karen Sutton, directora de operaciones, indica que NuVu tiene una filosofía de trabajo diferente a una escuela convencional, ya que anima a los estudiantes a que creen grupos con diferentes habilidades, a que sepan realizar tormentas de ideas, que investiguen y creen soluciones por cuenta propia, que sepan preparar prototipos haciendo constantes iteraciones, y que sepan presentarlos en público. Karen comenta que en NuVu la plantilla de especialistas no se denomina profesorado, sino animadores o coaches, porque en lugar de darles soluciones, los animadores facilitan a sus estudiantes a buscar aproximaciones (que no soluciones) por cuenta propia, y los estudiantes están obligados al final de cada día a documentar el proceso creativo en el estudio de fotografía, y a subirlo en el sitio web de cada proyecto.

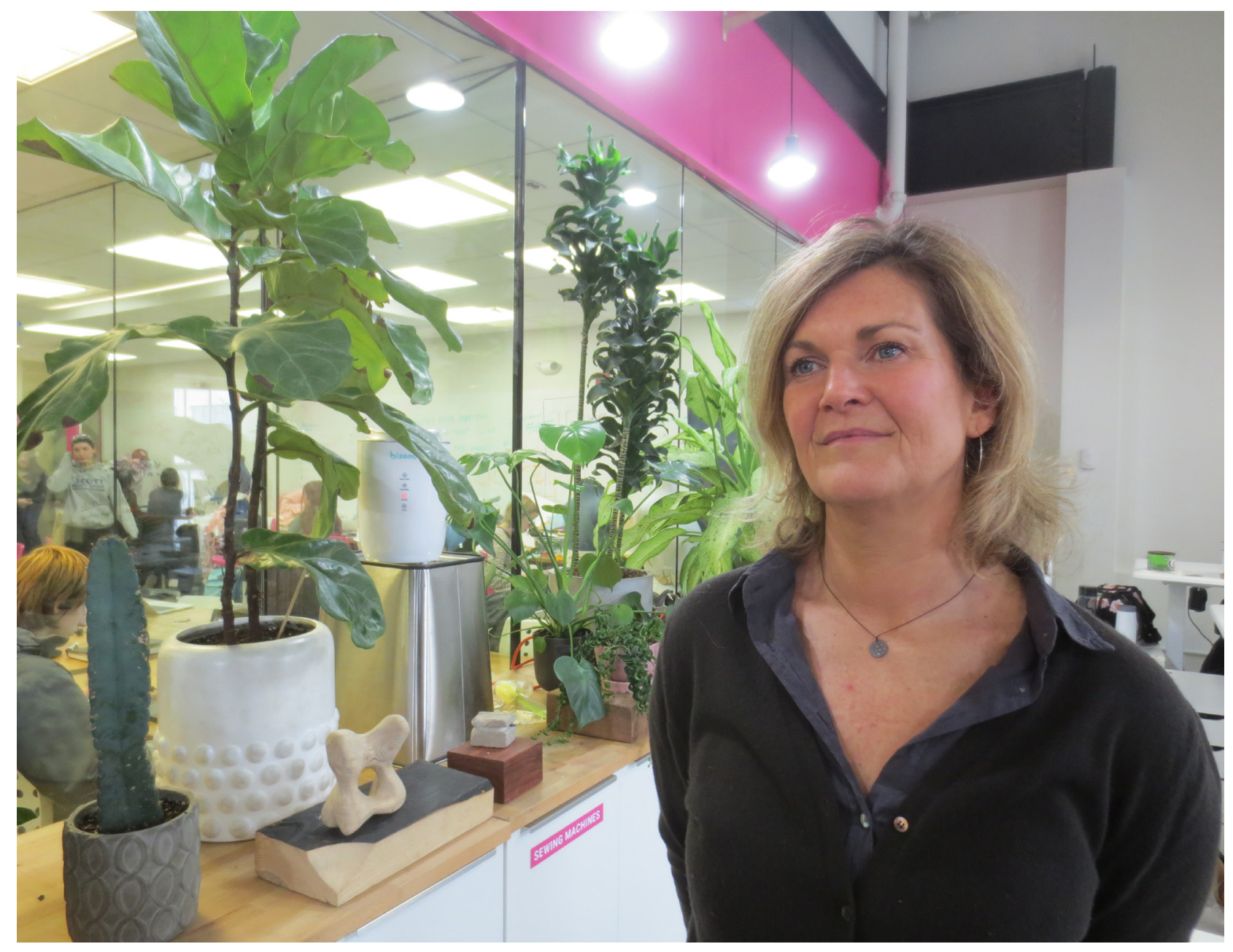

Fig. 2. Karen Sutton, NuVu, Cambridge. Fotografía de Jorge Paricio. 


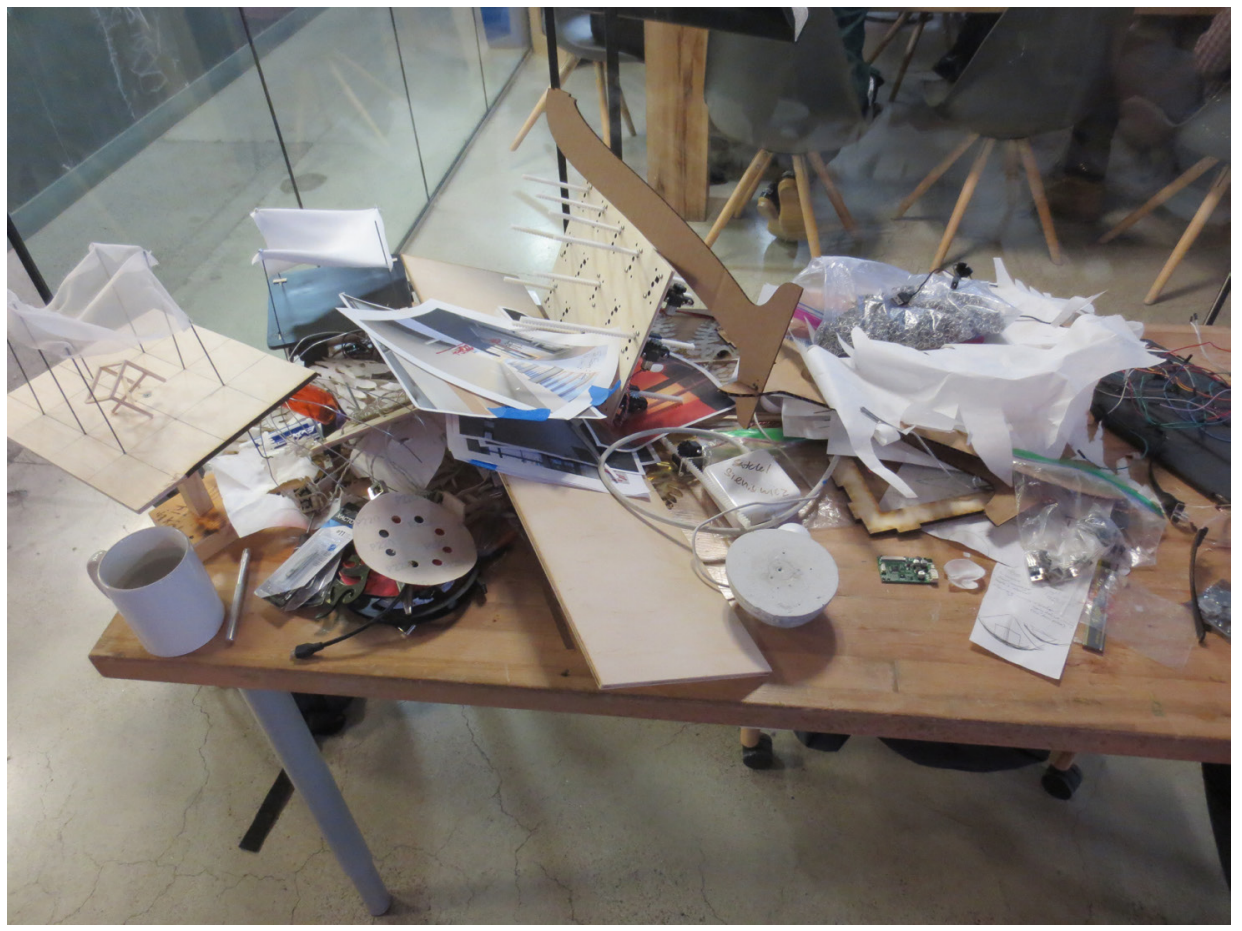

Fig. 3. Estación de trabajo en NuVu, Cambridge. Fotografía de Jorge Paricio.

Fruto de este ambiente de trabajo, los temas están constantemente renovándose y nunca duplican un mismo tema. Así, se han hecho talleres para imprimir comida en tres dimensiones, de arquitectura en la repostería, creación de películas documentales, realidad aumentada, arte interactivo, creación de Podcasts, etc.

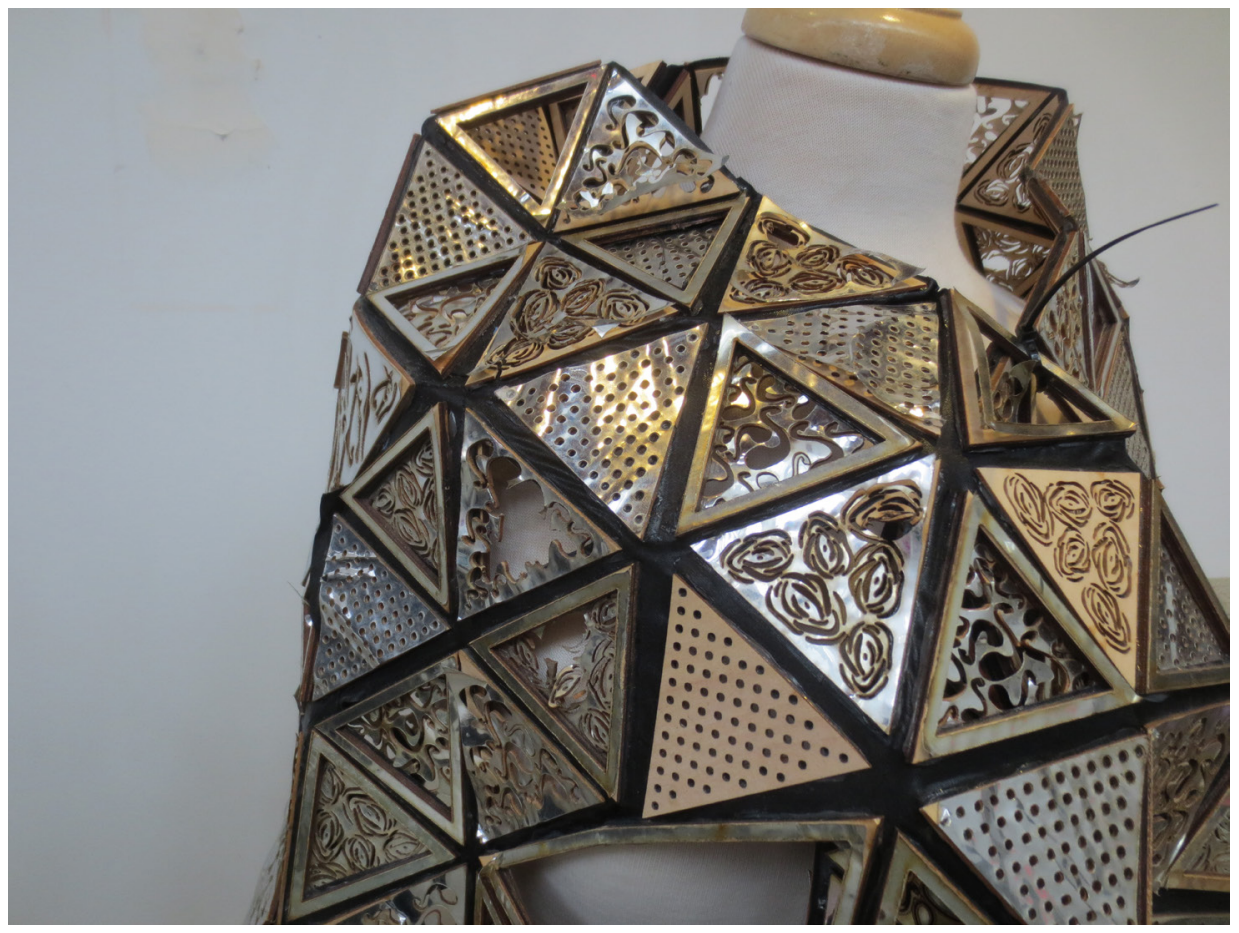

Fig. 4. Detalle de un prototipo de vestimenta, NuVu, Cambridge. Fotografía de Jorge Paricio. 


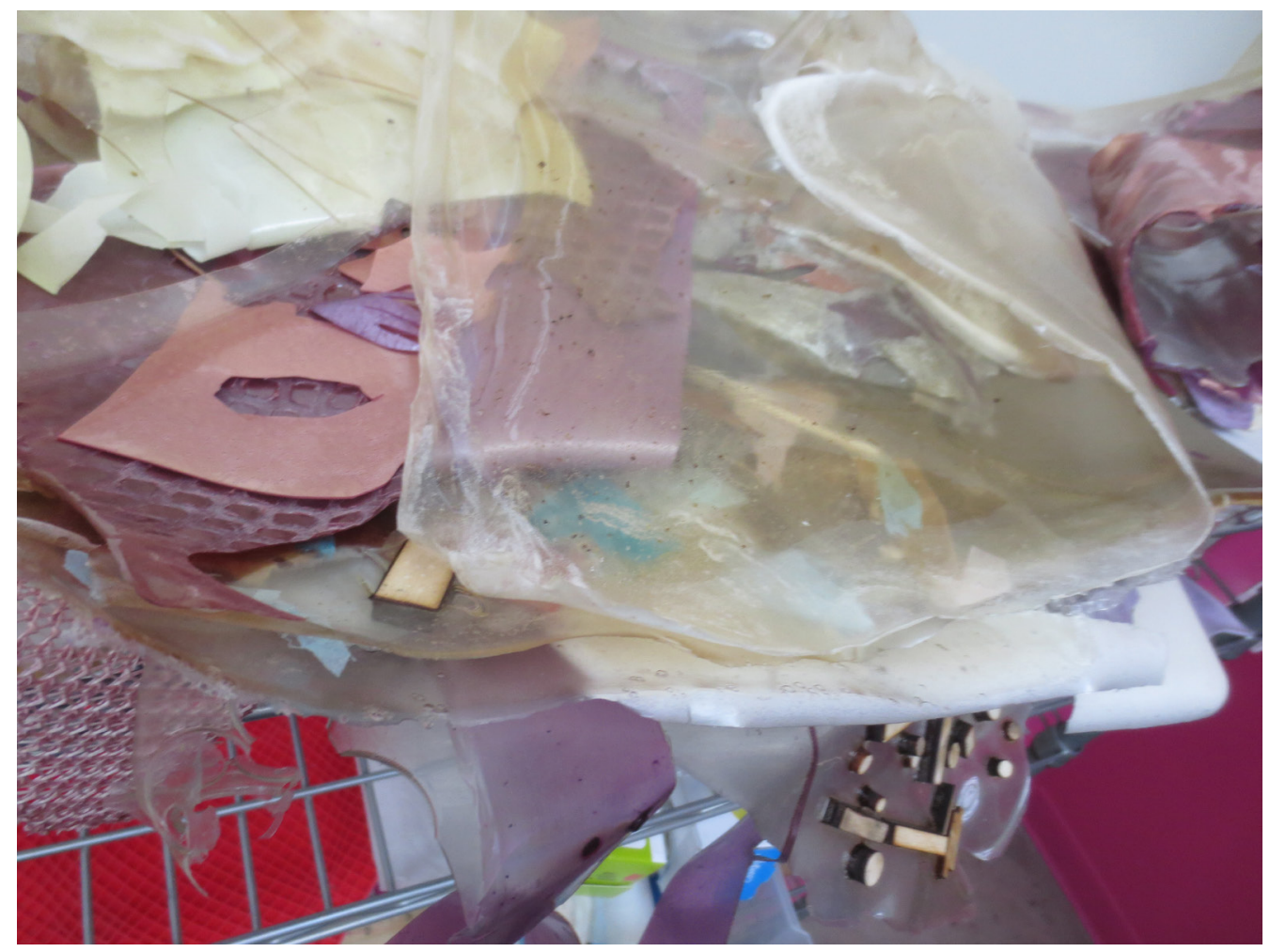

Fig. 5. Muestras de investigación de nuevos materiales, NuVu, Cambridge. Fotografía de Jorge Paricio.

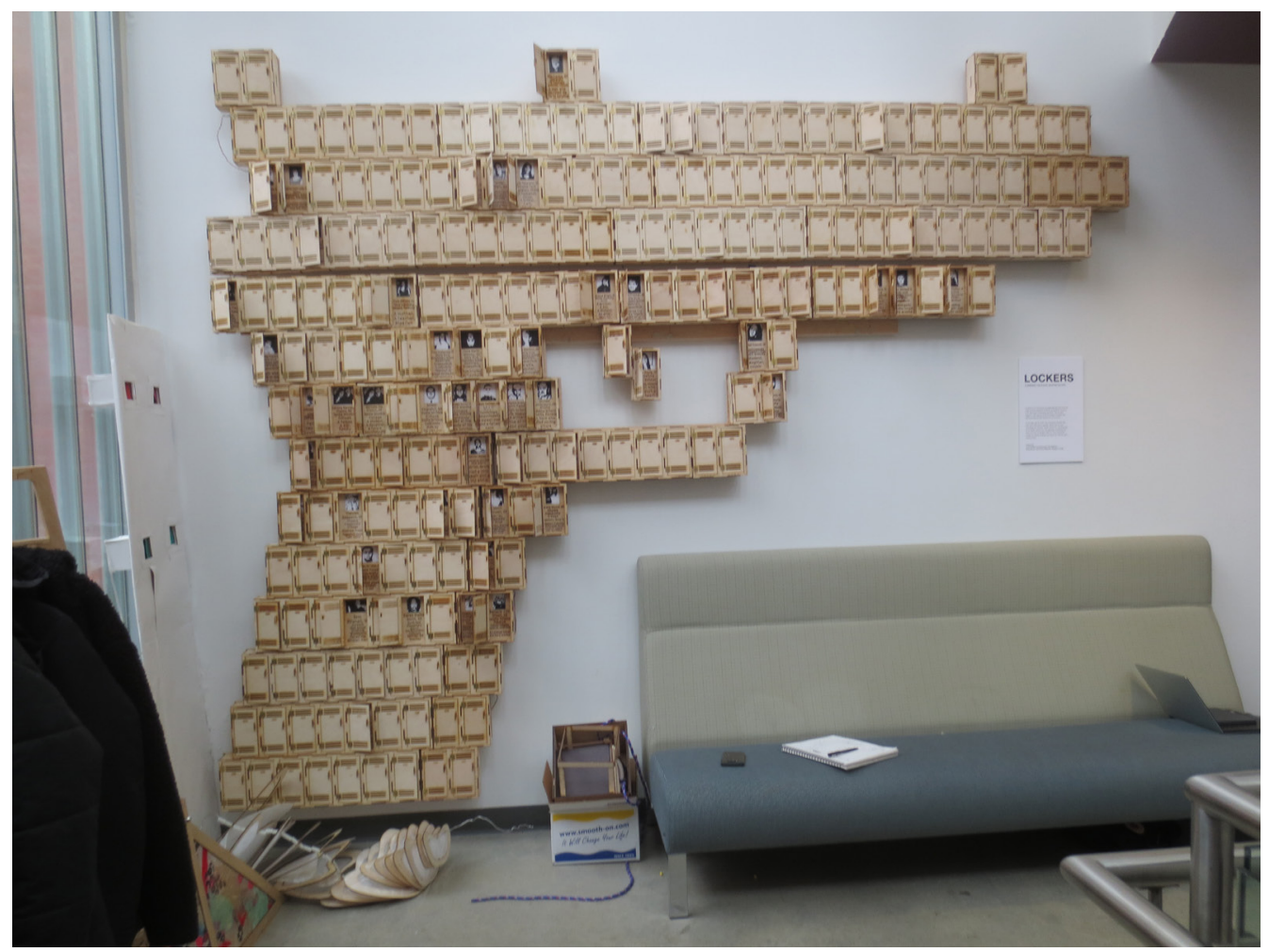

Fig. 6. Prototipo para la clase de arte conceptual, titulado "Lockers", una propuesta conmemorativa de las victimas por arma de fuego en las escuelas, NuVu, Cambridge. Fotografía de Jorge Paricio. 
Esta formación más acorde con el STEAM también está empezando en algunas escuelas de grado medio, museos y bibliotecas públicas. Este es el caso del Barrington Middle School en Rhode Island, que a solo diez minutos de RISD y Brown University tiene su propio laboratorio de fabricación, o Maker Lab, en donde se pueden crear oportunidades de colaboración entre estudiantes y las organizaciones, a través de proyectos reales. De igual manera, muchos estados están poniendo a disposición del público en general, laboratorios de fabricación a través de sus redes de bibliotecas públicas, en los que previa cita, se pueden utilizar impresoras en tres dimensiones, fresadoras de control numérico, cortadoras de vinilo y otros equipos.

La encargada de programas de enseñanza para jóvenes y escuelas del museo Isabella Stewart Gardner de Boston, Sara Egan, comenta que esta institución con más de 7.500 pinturas, esculturas y muebles, más de 1.500 libros únicos, y 7.000 objetos de gran variedad de culturas y países, ofrece programas que llenan el vacío educativo en arte que ya habíamos mencionado. Además de ofrecer programas educativos para sus visitantes, también tienen un acuerdo de colaboración con el sistema de enseñanza público de Boston, en donde ofrecen la oportunidad de enseñar a sus estudiantes a pensar de manera creativa, critical thinking, proponiendo un sistema en el que los estudiantes desarrollan su propia narrativa y aprenden a formular sus propias teorías y preguntas como: ¿qué crees que está pasando en esta pintura?, ¿qué más puedes encontrar? o ¿qué evidencias encuentras que consoliden tu teoría?

Igualmente, las universidades han visto el potencial de introducir el pensamiento creativo en el currículo de materias en los departamentos de educación contínua en los que también se enseña ajóvenes preuniversitarios. Así, estudiantes provenientes de muchas otras ciudades o países, acceden a clases a las que de otra manera no podrían tener acceso. En los últimos tres años, por ejemplo, China ha estado enviado a grupos selectos de estudiantes preuniversitarios a los Estados Unidos para recibir precisamente este tipo de clases, como introducción al diseño industrial a través de creación de prototipos, o clases de ilustración digital. RISD ha estado ofreciendo este tipo de clases como introducción al diseño de producto, en las que el autor de este artículo participa.

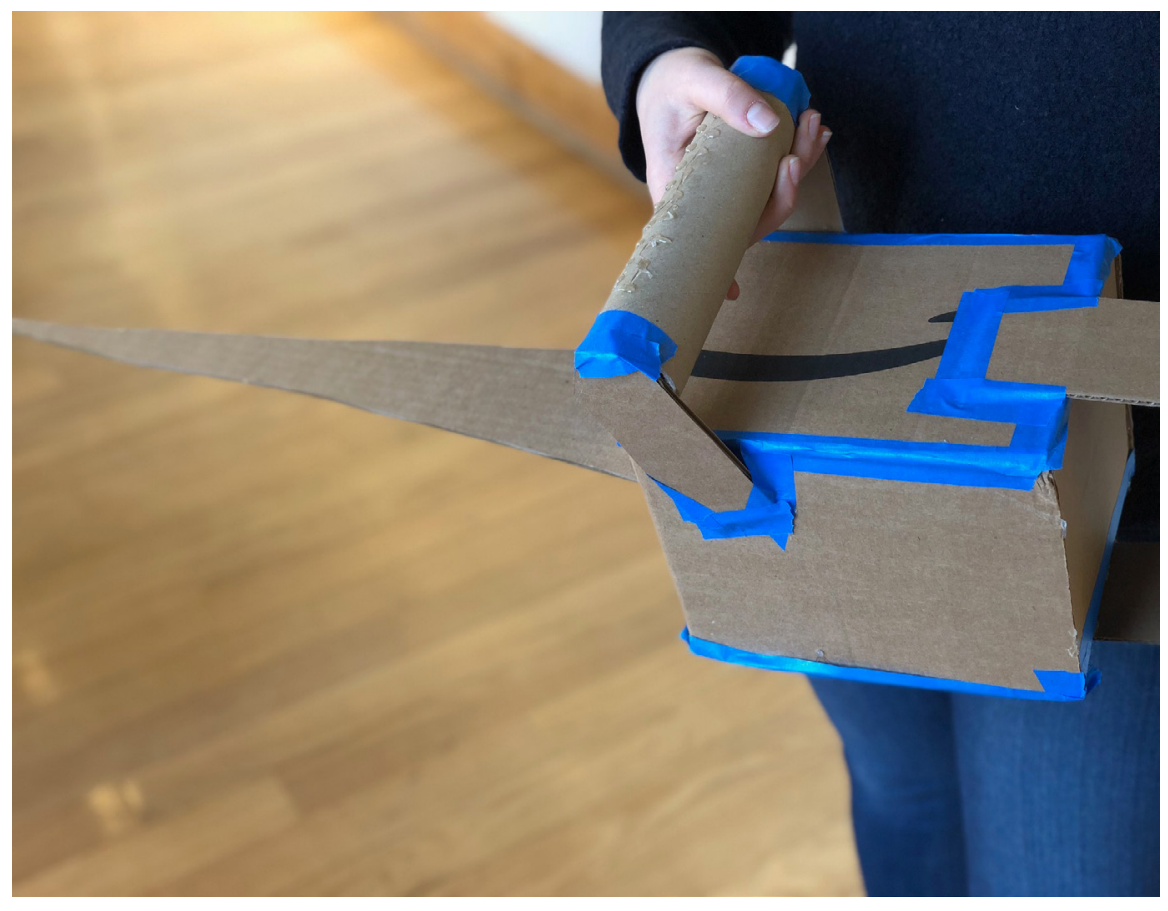

Fig. 7. Detalle de un prototipo para una maquina cortadora de setos, RISD, Providence. Fotografía de Tamar Shay Tannas. 
En definitiva, se ha comprobado que centros privados educativos, museos y otras instituciones se han dado cuenta del valor de fomentar una educación más global, en donde las materias consideradas científicas y de investigación ofrecen una ventana al arte y al diseño, como herramientas indispensables para desarrollar el potencial del pensamiento creativo en las mentes que todavía se están formando de cara al acceso a la universidad. 